\title{
Heavy alcohol use in patients on highly active antiretroviral therapy: What responses are needed?
}

\author{
C D Parry, ${ }^{1,2} \mathrm{PhD}$; C Kekwaletswe, ${ }^{3} \mathrm{PhD}$; P A Shuper, ${ }^{4} \mathrm{PhD} ;$ S Nkosi, ${ }^{3} \mathrm{MA} ;$ B J Myers, ${ }^{1,5} \mathrm{PhD} ; \mathbf{N}$ K Morojele, $, 3,6 \mathrm{PhD}$ \\ ${ }^{1}$ Alcohol, Tobacco and Other Drug Research Unit, South African Medical Research Council, Cape Town, South Africa \\ ${ }^{2}$ Department of Psychiatry, Faculty of Medicine and Health Sciences, Stellenbosch University, Tygerberg, Cape Town, South Africa \\ ${ }^{3}$ Alcohol, Tobacco and Other Drug Research Unit, South African Medical Research Council, Pretoria, South Africa \\ ${ }^{4}$ Centre for Addiction and Mental Health, Toronto, Canada; Dalla Lana School of Public Health, University of Toronto; Center for Health, \\ Intervention, and Prevention, University of Connecticut, Mansfield, USA \\ ${ }^{5}$ Department of Psychiatry and Mental Health, Faculty of Health Sciences, University of Cape Town, South Africa \\ ${ }^{6}$ School of Public Health, Faculty of Health Sciences, University of the Witwatersrand, Johannesburg, South Africa; School of Public Health \\ and Family Medicine, Faculty of Health Sciences, University of Cape Town, South Africa
}

Corresponding author: C D Parry (cparry@mrc.ac.za)

Background. Alcohol has a negative effect on antiretroviral therapy (ART) adherence and HIV treatment outcomes.

Method. As part of formative work for a project to test the efficacy of an alcohol-focused intervention to reduce alcohol consumption and improve HIV treatment outcomes, we investigated the extent of problem drinking among patients at ART clinics in Tshwane, South Africa (SA), using the Alcohol Use Disorders Identification Test (AUDIT).

Results. The finding that a third of drinkers reported hazardous drinking, roughly $10 \%$ reported harmful drinking, and a further $10 \%$ were possibly alcohol dependent replicates the findings of similar research in the Western Cape and Gauteng provinces of SA. It also points to the need for more routine screening of ART patients for problematic alcohol use.

Conclusion. The 10-item AUDIT may be too time consuming for health workers in busy ART clinics to administer and score, necessitating even briefer screening instruments for assessing hazardous and harmful drinking.

S Afr Med J 2016;106(6):567-568. DOI:10.7196/SAMJ.2016.v106i6.10639

The number of people on antiretroviral therapy (ART) in South Africa (SA) is the highest globally, accounting for over $20 \%$ of all persons on ART worldwide. ${ }^{[1]}$ This places an enormous burden on the health budget and on the human and other resources necessary to deliver ART to a greater proportion of HIV patients. However, increasing access to ART has made HIV a manageable, chronic condition. It is well established that hazardous or harmful alcohol use is causally linked to HIV disease progression. This occurs as a result of the effect of alcohol on ART adherence and on the immune system, and its interaction with ART and medications used to treat opportunistic infections. ${ }^{[2]}$ Yet little is known about the drinking behaviour of patients on ART in SA or how best to intervene.

In 2014, the South African Medical Research Council funded a randomised controlled trial (RCT) to determine the efficacy of an alcohol-focused intervention for improving adherence to ART and HIV treatment outcomes. ${ }^{[3]}$ As part of the formative work undertaken for this RCT, we investigated the extent of problem drinking among patients on ART seen at ART clinics in district hospitals in the Tshwane Metropolitan Municipality, Gauteng Province, SA.

\section{Methods}

Participants comprised patients who were screened to take part in the pilot study of the $\mathrm{RCT}^{[3]}$ as they waited for their clinic appointment. Patients were eligible to participate if they were at least 18 years old, lived in Tshwane or the general area served by the selected clinics, were HIV-positive, had been receiving ART for at least 3 months, were not currently on treatment for tuberculosis (TB), and were not currently enrolled in another study. The Alcohol Use Disorders
Identification Test (AUDIT) was then completed with consenting patients. ${ }^{[4]}$

The screener collected information on demographic characteristics, duration of ART, receipt of TB treatment, and alcohol use during the past 12 months. The 10-item $\operatorname{AUDIT}^{[4]}$ was administered to participants who reported any alcohol consumption in the past year. Responses were summed to yield a total score, with a possible range of 0 - 40. Higher total scores indicate a greater risk for problem drinking. The AUDIT cut-off scores of 8 - 15 for hazardous drinking, $16-19$ for harmful drinking and $\geq 20$ for possible alcohol dependence were used. ${ }^{[4]}$

\section{Results}

The sample comprised 196 participants who reported alcohol use in the past 12 months - one-third of those who reached this point in the screening process. Of these, 190 completed all items on the AUDIT. Roughly half were female (49.5\%). Among those who reported alcohol use in the past 12 months, a high proportion (46\%) were in the low-risk category. A third (33\%) of the participants were in the hazardous risk category, $11 \%$ were in the harmful risk category and $10 \%$ were in the possible dependence category. Overall, $69 \%$ of male drinkers and $39 \%$ of female drinkers scored in the highest three risk categories (i.e. total AUDIT score $\geq 8$ ). The mean total AUDIT score was significantly higher for men (11.4) than for women (7.9) $(t=-3.724 ; p<0.0001)$. One in five female drinkers (21\%) reported heavy episodic drinking at least once a month, compared with a third (32\%) of male drinkers (heavy episodic drinking being defined as four or more drinks for women and six or more drinks for men). 


\section{Discussion}

Although the sample size was relatively small and excluded individuals who refused screening or reported no drinking in the past year, the findings broadly align with those of Kader et al. ${ }^{[5]}$ who found that $91 \%$ of male and $89 \%$ of female drinkers (most of whom were receiving ART) attending eight HIV clinics in Cape Town reported hazardous or harmful alcohol consumption when screened with the AUDIT. A similar study conducted in Tshwane in two HIV clinics among patients on ART found slightly lower corresponding figures of $75 \%$ for male and $55 \%$ for female drinkers, but like the findings of Kader et al. ${ }^{[5]}$ these are somewhat higher than ours reported above.

The high proportions of men and women who drink heavily is of grave concern given the associated risk for reduced ART adherence, ${ }^{[6]}$ forward transmission of HIV, and other health problems. ${ }^{[7]}$ Given the high levels of problematic drinking that were evident among patients on ART in Tshwane, we agree with recommendations made previously ${ }^{[2,5]}$ that there should be more screening of patients attending HIV clinics for problematic alcohol use as a prelude to implementing brief interventions to reduce the negative impact of drinking on HIV treatment outcome, or where necessary referring HIV patients to more intensive treatment. However, the $2-4$ minutes typically taken to administer and score the AUDIT ${ }^{[4]}$ is likely to serve as a barrier to its routine use in primary care settings, and as a result further research is also needed to assess the possibility of using briefer screening instruments in primary care settings.

Acknowledgements. We acknowledge funding from the South African Medical Research Council (Flagship Research) for the study referred to and for our time in preparing this article.

1. UNAIDS. How AIDS Changed Everything - MDG6: 15 Years, 15 Lessons of Home from the AIDS Response. Geneva: UNAIDS, 2015.

2. Schneider M, Neuman M, Chersich, M, Parry, C. Alcohol and antiretroviral therapy - a lethal cocktail. J AIDS Clin Res 2012;S1:005. DOI:10.4172/2155-6113.S1-005

3. Parry $\mathrm{CDH}$, Morojele NK, Myers BJ, et al. Efficacy of an alcohol-focused intervention for improving adherence to antiretroviral therapy (ART) and HIV treatment outcomes - a randomised controlled trial protocol. BMC Infect Dis 2014:14:500. DOI:10.1186/1471-2334-14-500

4. Babor TF, Higgins-Biddle JC, Saunders JB, Monteiro MG. The Alcohol Use Disorders Identification Test: Guidelines for Use in Primary Care. Geneva: World Health Organization, Department of Mental Health and Substance Abuse Dependence, 2001. http://whqlibdoc.who.int/hq/2001/WHO_MSD_ MSB_01.6a.pdf (accessed 6 May 2016).

5. Kader R, Seedat S, Govender R, Parry CD. Hazardous and harmful use of alcohol and/or other drugs and health status among South African patients attending HIV clinics. AIDS Behav 2014;18(3):525534. DOI:10.1007/s10461-013-0587-9

6. Hendershot CS, Stoner SA, Pantalone DW, Simoni JM. Alcohol use and antiretroviral adherence: Review and meta-analysis. J Acquir Immune Defic Syndr 2009;52:180-202. DOI:10.1097/ QAI.0b013e3181b18b6e

7. Rehm J, Baliunas D, Borges GLG, et al. The relation between different dimensions of alcohol consumption and burden of disease - an overview. Addiction 2010;105(5):817-843. DOI:10.1111/ j.1360-0443.2010.02899.x

Accepted 4 April 2016 